\title{
Enfermedad de Parkinson: fisiopatologia, diagnóstico y tratamiento
}

\section{Parkinson disease: pathophysiology, diagnosis and treatment}

\author{
Daniel-S Marín-M ${ }^{1,2}$; Hans Carmona-V ${ }^{1,3}$; Melissa Ibarra-Q1; Manuela Gámez-C ${ }^{2}$
}

Forma de citar: Marín D, Carmona H, Ibarra M, Gámez M. Enfermedad de Parkinson: fisiopatología, diagnóstico y tratamiento. Rev Univ Ind Santander Salud. 2018; 50(1): 79-92. doi: http://dx.doi.org/10.18273/revsal.v50n1-2018008 (c) (1)

\section{Resumen}

La enfermedad de Parkinson es la segunda causa de enfermedad neurodegenerativa crónica progresiva, tiene una alta prevalencia e incidencia, genera un alto impacto en la calidad de vida de los pacientes e importantes costos en su atención. La enfermedad de Parkinson se desarrolla por la degeneración de las neuronas dopaminérgicas en la sustancia nigra pars compacta, lo que se manifiesta en la aparición de síntomas motores como la bradicinesia, temblor de reposo, rigidez e inestabilidad postural; así como también de síntomas no motores como alteraciones gastrointestinales, del sueño, autonómicas, cognitivas, entre otras, que reflejan el compromiso de diferentes vías no dopaminérgicas. El diagnóstico se apoya en sus manifestaciones clínicas más características y excluye otras causas de parkinsonismo. El tratamiento farmacológico busca controlar los síntomas motores y no motores, los cuales empeoran por la historia natural de la enfermedad o se acompañan de complicaciones debidas a la terapia, haciendo necesarias otras intervenciones como la estimulación cerebral profunda.

Palabras clave: Enfermedad de Parkinson; Trastornos del movimiento; Temblor; Levodopa; Estimulación cerebral profunda.

\begin{abstract}
Parkinson's disease is the second cause of progressive chronic neurodegenerative disease, it has a high prevalence and incidence, generates a high impact on the quality of life of patients and significant costs due to its healthcare. Parkinson's disease is developed due to the degeneration of dopaminergic neurons in the substance nigra pars compacta, which is manifested in the appearance of motor symptoms such as bradykinesia, rest tremor, rigidity and postural instability; as well as non-motor symptoms such as gastrointestinal, sleep, autonomic, cognitive alterations, among others, reflecting the impairment of different non-dopaminergic pathways. The diagnosis is based on its most frequent clinical manifestations and the exclusion of other causes of parkinsonism. The pharmacological treatment seeks to control motor and non-motor symptoms, which are worsened by the natural history of the disease or are accompanied by side effects induced by pharmacotherapy, making necessary other approaches such as deep brain stimulation.
\end{abstract}

Keywords: Parkinson disease; Movement disorders; Tremor; Levodopa; Deep brain stimulation.

1. Grupo de Investigación "Applied Neuroscience”, Neurocentro. Risaralda, Colombia.

2. Universidad Tecnológica de Pereira. Risaralda, Colombia.

3. Universidad Autónoma de las Américas-Pereira, Risaralda, Colombia.

Correspondencia: Daniel Stiven Marín Medina. Dirección: Carrera 27\#10-02 Álamos, U. Tecnológica de Pereira, Edificio 14, Facultad de Ciencias de la Salud, Piso 3, Ciencias comunitarias. Correo electrónico: cercaylejos@utp.edu.co. Teléfono: +57 63154506490. 


\section{Introducción}

La enfermedad de Parkinson (EP) es el tipo de parkinsonismo más frecuente, cerca de 10 millones de personas en el mundo la padecen, afecta al $1 \%$ de la población mayor de 60 años y 4-5\% de los mayores de 85 años ${ }^{1-3}$. La prevalencia global de EP varía entre $100-300$ por cada 100.000 habitantes $^{4}$ y la incidencia está entre 1,5-22 para todas las edades ${ }^{4-6}$, aunque ambas aumentan de forma dramática conforme avanza la edad ${ }^{4,7-9}$, estabilizándose a los 80 años $^{6}$. El estudio EPINEURO encontró que existen 4,7 afectados por cada mil habitantes en Colombia ${ }^{10}$. La EP se presenta con mayor frecuencia en hombres que en mujeres, en una relación de 1,5:111.

La media de supervivencia en pacientes con EP es de 11-15,8 años ${ }^{12-14}$, siendo la neumonía (11-28\%), enfermedades cardiovasculares (12-19\%) y cáncer $(12-14 \%)$ las principales causas de muerte ${ }^{13,15-17}$. En los pacientes de 25-39 años de edad la expectativa de vida alcanza los 38 años, mientras que en los mayores de 65 años baja a cinco años ${ }^{18}$. Los costos médicos directos por consulta externa (horas de personal de salud, insumos, medicamentos, entre otros) avalúan un promedio de USD 485,74 al mes por paciente ${ }^{19}$, que podría alcanzar hasta 5.993 euros al año ${ }^{20}$. La calidad de vida del paciente con EP se deteriora progresivamente y crea una gran carga para sus cuidadores ${ }^{21,22}$, la cual aumenta por los trastornos del sueño, de la cognición y la conducta, la depresión, ansiedad, duración de la enfermedad y la discapacidad que genera la $\mathrm{EP}^{23-26}$.

El objetivo de este artículo es realizar una revisión narrativa de los principales aspectos implicados en la EP, las manifestaciones clínicas que llevan a su diagnóstico y los posibles tratamientos.

\section{Patogenia y fisiopatología}

Los pesticidas, la residencia en el campo y la agricultura se asocian fuertemente a EP. Una alta ingesta de hierro, anemia crónica, trauma craneoencefálico grave y trabajos de alta complejidad cognitiva también se han visto asociados ${ }^{27-30}$. Los principales factores protectores son la hiperuricemia, tabaquismo y café ${ }^{4,29,31}$, mientras que la vitamina E, alcoholismo, té, AINES y el ejercicio físico vigoroso tienen una asociación menor ${ }^{27}$. Estos factores sumados a factores genéticos podrían ser la explicación de la mayoría de los casos de EP, dado que las causas genéticas por sí solas contribuyen en un $5-10 \%$ de los $\operatorname{casos}^{32,33}$, lo cual podría explicar la aparición de la enfermedad antes de los 50 años $^{9,34}$.
El origen del proceso de degeneración que sufren las neuronas dopaminérgicas podría estar en la disfunción mitocondrial, agregación de $\alpha$-sinucleina, alteración de la autofagia, estrés del retículo endoplasmático (RE) o la desregulación de la homeostasis intracelular de calcio ${ }^{35}$. La disfunción mitocondrial se ha evidenciado en la disminución de la actividad del complejo I de la cadena transportadora de electrones ${ }^{36,37}$, un mecanismo similar al mediado por el MPTP que es una toxina ambiental que causa parkinsonismo ${ }^{38}$ y muerte de neuronas dopaminérgicas de la SN pars compacta ${ }^{39}$; además, las mutaciones de genes asociados a EP como la parkina ${ }^{40}$ y PINK $141^{41}$ generan propensión al daño por estrés oxidativo y deterioran la homeostasis mitocondrial ${ }^{42}$. La $\alpha$-sinucleina estáinvolucrada en el tráfico, exocitosis delas vesículas sinápticas y liberación de neurotransmisores ${ }^{43}$; las mutaciones en el gen SNCA que codifica la proteína $\alpha$-sinucleina producen un mal plegamiento y agregación de esta proteína con la consecuente formación de fibrillas similares a amiloides, conocidos como cuerpos de Lewy ${ }^{44,45}$, que se acumulan y podrían tener un efecto neurotóxico ${ }^{35,46,47}$. Las alteraciones en la autofagia también justificarían las inclusiones intracelulares de proteínas como la $\alpha$-sinucleina ${ }^{48,49}$; además, la sobreexpresión de la $\alpha$-sinucleina inhibe el mecanismo de autofagia al inhibir la Rabla, proteína esencial para la formación del autofagosoma ${ }^{50} \mathrm{y}$ hace que se bloquee su propia degradación ${ }^{51}$. Por último, la desregulación de la homeostasis del calcio origina la activación de enzimas que desencadenan una cascada apoptótica en las neuronas ${ }^{52}$.

Las neuronas dopaminérgicas de la sustancia negra (SN) pars compacta ${ }^{53,54}$ hacen parte de la vía nigraestriatal que se proyecta hacia los ganglios basales, en donde regulan la actividad de neuronas estriatales cuyas eferencias modulan la actividad del tálamo de forma directa o indirecta. La vía indirecta normalmente es inhibida por la liberación de dopamina en los receptores D2 de las neuronas estriatales (núcleo caudado y putamen), esta inhibición se pierde en la EP por la deficiencia de dopamina, haciendo que se desinhiban las neuronas estriatales, las cuales a su vez inhiben el globo pálido externo ( $\mathrm{GPe}$ ) que normalmente inhibe el núcleo subtalámico (NST), dando como resultado la desinhibición de este último. EL NST envía proyecciones glutamatérgicas hacia la SN pars reticulata y al globo pálido interno (GPi), desde donde se inhibe los núcleos talámicos que se proyectan hacia la corteza cerebral, lo que en últimas resulta en una disminución de la actividad locomotora y la consiguiente bradicinesia ${ }^{55-57}$, Figura 1. Por otra parte, la vía directa normalmente es excitada por la liberación de dopamina 
sobre los receptores D1 de las neuronas estriatales, esta excitación se pierde en la EP dando lugar a la inhibición de las neuronas estriatales, las cuales a su vez inhiben el GPi y la SN pars reticulata que normalmente inhiben la actividad del tálamo, por lo que éste último queda libre para enviar impulsos excitatorios a la corteza motora y generando así la actividad motora en forma de temblor ${ }^{55-57}$, Figura 2.

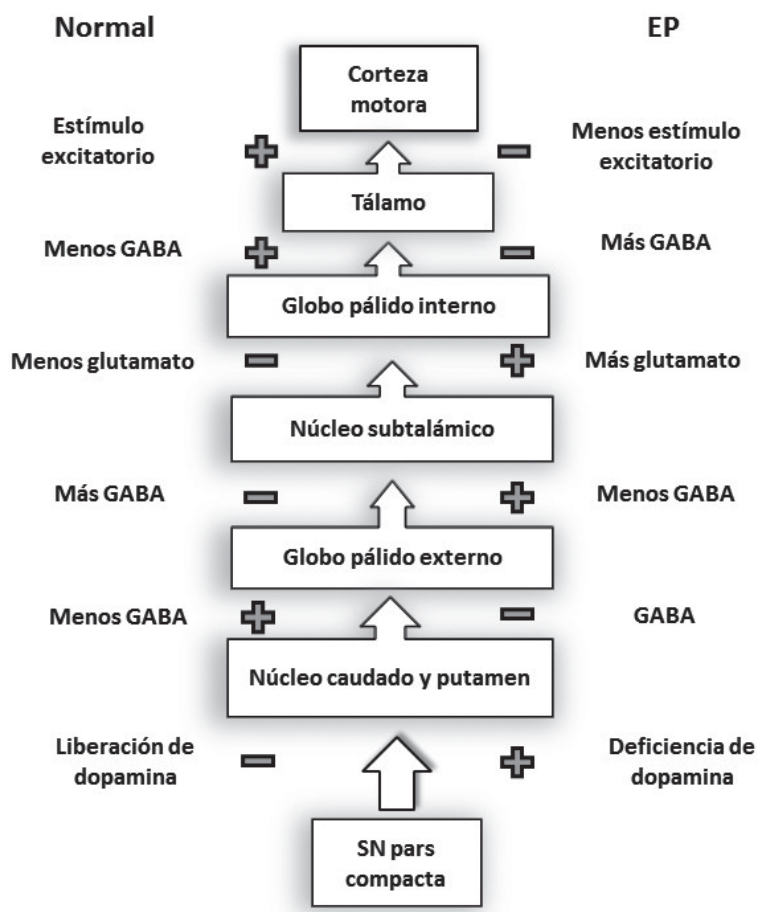

Figura 1. Vía indirecta. EP: enfermedad de Parkinson; SN: sustancia nigra.

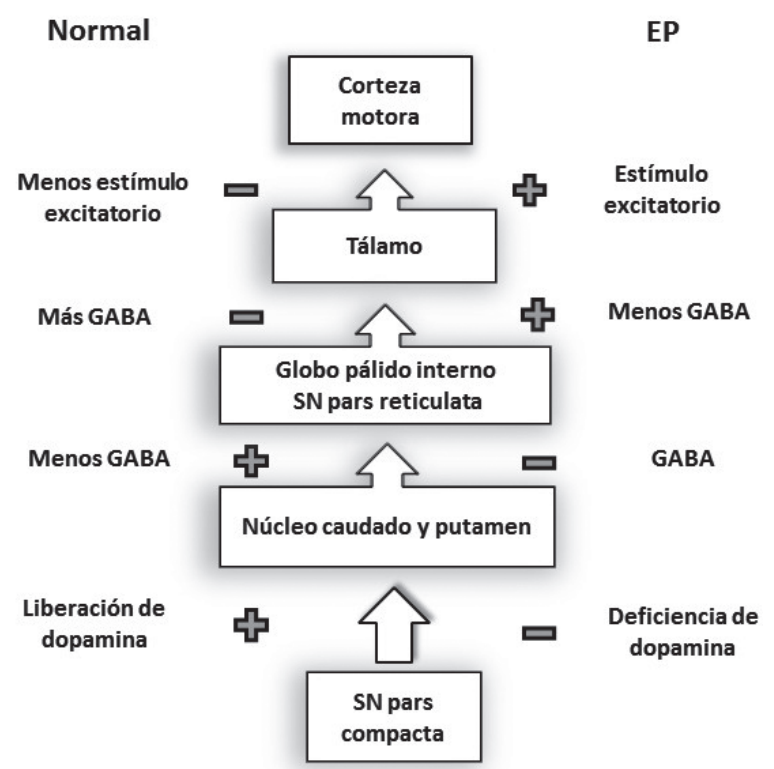

Figura 2. Vía directa. EP: enfermedad de Parkinson; SN: sustancia nigra.

\section{Manifestaciones clínicas y diagnóstico}

El diagnóstico de la EP es eminentemente clínico. Los criterios más ampliamente aceptados fueron introducidos por la UK Parkinson Disease Society - Brain Bank, los cuales incluyen cuatro signos cardinales: bradicinesia-acinesia, temblor en reposo, rigidez e inestabilidad postural ${ }^{58,59}$, Tabla 1.

Tabla 1. Criterios clínicos para el diagnóstico de EP.

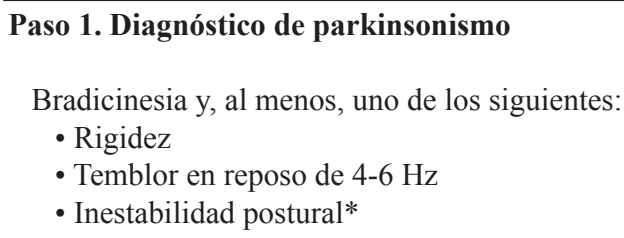

Paso 2. Excluir otras causas de parkinsonismo

\section{Paso 3. Criterios que apoyan el diagnóstico de EP}

Al menos tres de los siguientes:

- Inicio unilateral.

- Temblor de reposo

- Trastorno progresivo

- Afectación asimétrica con mayor afectación unilateral desde el inicio

- Excelente respuesta a la levodopa

- Corea inducida por levodopa

- Respuesta a la levodopa durante 5 años

- Curso clínico superior a 10 años

\footnotetext{
* No causada por alteración visual, vestibular, cerebelosa o disfunción propioceptiva
}

La bradicinesia es la característica más frecuente en la EP. Esta se refiere a la lentificación progresiva de los movimientos y abarca las dificultades en la planeación, iniciación y ejecución de tareas que requieran movimientos secuenciales y simultáneos ${ }^{60,61}$. Inicialmente el paciente percibe mayor lentitud en sus reacciones o al realizar tareas de la vida diaria, en especial aquellas en las que se vea involucrada la motricidad fina ${ }^{62}$. Es posible distinguirla de otras condiciones con lentificación de los movimientos como la depresión, espasticidad o la paresia, haciendo pruebas que impliquen algún movimiento repetitivo que se haga lo más rápido y amplio que el paciente sea capaz (abrir y cerrar la mano, golpear el índice con el pulgar o marcar el paso con el pie en el piso), las cuales en el paciente con EP mostrarán una lentificación o disminución de la amplitud progresiva hasta llegar a detenerse por completo $^{63}$. La bradicinesia también se manifiesta en disartria con monofonía e hipofonía, hipomimia (poca expresión facial, conocida como facies de jugador de poker), sialorrea por dificultad para tragar, pérdida de 
movimientos espontáneos, micrografía, disminución del parpadeo y del braceo durante la marcha ${ }^{62-64}$.

El temblor en reposo de los pacientes con EP suele ser inicialmente unilateral, con una frecuencia de 3-6 $\mathrm{Hz}$, amplitud variable, cesa al realizar movimientos voluntarios o durante el sueño, y afecta generalmente la parte distal de la extremidad (signo del contador de monedas, movimiento repetitivo de frotamiento del pulgar sobre el índice), aunque otras partes del cuerpo como los labios, la barbilla, la mandíbula y las piernas también pueden afectarse, sin llegar a comprometer el cuello, la cabeza o la voz ${ }^{62,63}$. En algunos pacientes es posible encontrar temblor postural, conocido como temblor reemergente, el cual aparece poco después de que el paciente extiende la mano ${ }^{63,65}$.

La rigidez se evidencia en el aumento de la resistencia durante los diferentes movimientos pasivos de la extremidad (flexión, extensión o rotación alrededor de una articulación), conocida como signo de la rueda dentada y puede ser reforzada mediante la maniobra de Froment (movimiento de la extremidad contralateral a la examinada ${ }^{66}$. La rigidez puede aparecer tanto proximal como distal y está asociada con dolor, especialmente de hombro. La rigidez de cuello y tronco lleva a una posición flexionada que cuando es extrema se conoce como camptocormia ${ }^{62,63}$.

La inestabilidad postural aparece tardíamente en la EP, es causada por la pérdida de los reflejos posturales y contribuye en gran parte al riesgo de caídas en los pacientes con $\mathrm{EP}^{67}$. Ésta se evalúa con una prueba en la cual el paciente con EP toma más de dos pasos hacia atrás o adelante, o no responde adecuadamente con el tono postural luego de hacerle una tracción rápida de los hombros hacia atrás o adelante ${ }^{62,63}$.

Un grupo de síntomas en la EP no son motores, estos se han tratado de explicar a través de la disfunción de neuronas no dopaminérgicas que conlleva a la degeneración del bulbo olfatorio, alteraciones del centro del sueño en el tronco cerebral y de los núcleos medulares que controlan las funciones autonómicas, acumulación de cuerpos de Lewy en las estructuras límbicas y la neocorteza ${ }^{68,69}$. Estas alteraciones pueden aparecer incluso antes que los síntomas motores, ocasionando con más frecuencia manifestaciones como la constipación, hiposmia/anosmia, trastorno de la conducta durante el sueño REM y depresión, aunque también se ha asociado en menor medida el síndrome de piernas inquietas, apatía, fatiga y ansiedad ${ }^{70}$. Otros trastornos del sueño incluyen el insomnio, somnolencia diurna excesiva y alteración del ciclo vigília-sueño ${ }^{71}$. La disfunción autonómica se refleja en alteraciones de la micción, sudoración, hipotensión ortostática, disfunción sexual y xerostomía ${ }^{72}$. Los síntomas gastrointestinales abarcan la ageusia, disfagia, reflujo, vómitos e incontinencia ${ }^{73}$. Los síntomas neuropsiquiátrios incluyen anhedonia, alucinaciones, ideas delirantes, conducta obsesiva, confusión, delirio y ataques de pánico ${ }^{74}$. Algunos pacientes pueden presentar manifestaciones sensitivas como dolor y parestesias ${ }^{75}$.

En el paciente con síntomas parkinsonianos se debe buscar otras causas diferentes a la EP cuando hay historia de accidente cerebrovascular a repetición, daño cerebral repetido, uso de drogas antipsicóticas o antidopaminérgicas, encefalitis o crisis oculógiras no tratadas, tumor cerebral o hidrocefalia demostradas por neuroimagen, exposición a una neurotoxina conocida, cuando hay más de un miembro de la familia afectado, si ha habido remisión clínica prolongada, respuesta negativa a dosis elevada de levodopa, afectación estrictamente unilateral después de tres años del comienzo de la clínica, u otros síntomas neurológicos como: parálisis supranuclear de la mirada, signos cerebelosos, deterioro autonómico severo en fases tempranas, signo de Babinski, demencia o trastornos del lenguaje y memoria severos en fases tempranas ${ }^{58,59}$.

Luego de diagnosticar la EP el siguiente paso es clasificar a los pacientes según la escala de Hoehn and Yahr, la cual valora la progresión, gravedad de la enfermedad y puede ser usada para evaluar la mejoría con el tratamiento, aunque solo tiene en cuenta los síntomas motores ${ }^{76}$, Tabla 2.

Tabla 2. Escala de Hoenh y Yarh modificada (152).

Estadio 1 Síntomas unilaterales solamente

Estadio 1,5 Síntomas unilaterales y axiales

Estadio 2 Síntomas bilaterales, sin alteraciones de equilibrio Estadio 2,5 $\begin{aligned} & \text { Síntomas bilaterales leves, con mejoría en pull } \\ & \text { test* }\end{aligned}$

Enfermedad bilateral leve a moderada

Estadio 3 con inestabilidad postural; físicamente independiente.

Estadio 4 Incapacidad grave, aún capaz de caminar o permanecer de pie sin ayuda.

Estadio 5 Permanece en silla de ruedas o en cama si no tiene ayuda.

* Test de retropulsión o anteropulsión. 


\section{Tratamiento de los síntomas motores}

Existen diferentes opciones farmacológicas disponibles para el tratamiento inicial de la EP, las cuales dependen del compromiso funcional del paciente en las actividades laborales o cotidianas y la percepción de éste frente a su enfermedad ${ }^{77}$. En pacientes sin compromiso funcional se puede considerar los inhibidores de la monoaminooxidasa tipo B (iMAO-B), amantadina o anticolinérgicos ${ }^{78}$. En los pacientes con compromiso funcional se debe tener en cuenta la edad y estado cognitivo: en aquellos de mayor edad ( $\geq 65$ años) y con deterioro cognitivo se recomienda iniciar con levodopa, vigilando la aparición de alucinaciones y trastornos de la conducta ${ }^{79}$; en los pacientes de mayor edad sin deterioro cognitivo se puede emplear levodopa asociada a otros fármacos antiparkinsonianos; y en los pacientes jóvenes $(<65$ años) se debe evitar la levodopa como terapia inicial o usarse a dosis bajas, prefiriendo los agonistas dopaminérgicos ${ }^{80-83}$. A pesar de un buen manejo y del control de los síntomas de la EP durante los primeros años de iniciado el tratamiento farmacológico, muchos pacientes desarrollan complicaciones a largo plazo y es por esta razón que el tratamiento de las fases tardías de la EP requiere de diferentes estrategias para el manejo de los síntomas motores y no motores ${ }^{84}$.

\section{Levodopa}

La levodopa es el fármaco más importante utilizado en el tratamiento de la EP, es un precursor de la dopamina que no sufre la rápida degradación de ésta en el tracto gastrointestinal pero que sí se degrada en la circulación sistémica, por lo cual se prescribe junto a inhibidores de la descarboxilasa (carbidopa o benserazida), logrando disminuir las dosis necesarias y los efectos secundarios al permitir una mayor distribución en el sistema nervioso central ${ }^{85}$. Se inicia con una dosis baja usando tabletas de $100 \mathrm{mg}$ levodopa y $25 \mathrm{mg}$ carbidopa, se indica la mitad de la tableta dos o tres veces al día acompañada de las comidas, luego de lo cual se puede titular durante varias semanas hasta alcanzar la tableta completa tres veces al día, a esta dosis una gran parte de los pacientes mostrarán una buena respuesta con una dosis diaria de 300-600 mg dividida tres o cuatro veces al día ${ }^{85,86}$. Las dosis altas deben ser evitadas porque aumentan el riesgo de discinesias, teniendo en cuenta que la ausencia de respuesta a altas dosis obliga a descartar otras causas de parkinsonismo ${ }^{62,87}$. Los efectos secundarios más comunes son las náuseas, vómitos, cefalea y en pacientes de mayor edad puede aparecer delirio, agitación, alucinaciones o psicosis ${ }^{85}$.
Más de la mitad de los pacientes con EP desarrollan complicaciones asociadas al tratamiento con levodopa luego de 5-10 años de iniciado el tratamiento ${ }^{88,89}$. Estas complicaciones incluyen: fluctuaciones motoras (fenómeno de desgaste o "wearing-off"), discinesias y otras complicaciones motoras ${ }^{90,91}$, las cuales pueden ser causados por la alteración de las variaciones fisiológicas de los niveles plasmáticos de dopamina ocasionada por la EP o también pueden ser atribuidas al estrés oxidativo y neurodegeneración generados por la levodopa, aunque esto último aún no ha sido demostrada in vivo ${ }^{92-94}$.

\section{Agonistas dopaminérgicos}

Este grupo de medicamentos genera una estimulación directa sobre los receptores de dopamina, destacándose entre ellos el ropinirol, pramipexol y rotigotina ${ }^{95}$. Los agonistas de la dopamina están asociados a menos fluctuaciones motoras y discinesias en comparación con levodopa y se los considera ahorradores de levodopa porque retrasan la necesidad de emplear este medicamento y por lo tanto la aparición de discinesias asociadas al tratamiento ${ }^{96-98}$. En la EP temprana los agonistas de la dopamina tienen una eficacia comparable a levodopa, pero en las fases más avanzadas esta eficacia disminuye, por lo cual solo se usan en monoterapia en los pacientes más jóvenes que no tienen compromiso cognitivo o en los pacientes mayores sin compromiso cognitivo, y es necesario asociarlos a levodopa cuando ya no logren un buen control de los síntomas ${ }^{80-83}$, evitando emplearlos en los pacientes con deterioro cognitivo quienes podrían ser más propensos a las alucinaciones u otros de los efectos adversos de los agonistas de la dopamina: somnolencia excesiva, hipotensión ortostática, edema y trastornos del control de los impulsos (juego patológico, compras, uso de internet, hipersexualidad $)^{98-100}$. Las dosis iniciales deben ser bajas y aumentarse progresivamente, pramipexole se inicia con $0,125 \mathrm{mg}$ tres veces al día hasta llegar a 1,5-4.5 mg/día; la rotigotina se inicia en parches de $2 \mathrm{mg}$ para 24 horas hasta $16 \mathrm{mg}$ en 24 horas; mientras que el ropinirol se empieza con $0,25 \mathrm{mg}$ tres veces al día hasta llegar a $3 \mathrm{mg}^{77}$.

\section{Inhibidores de la monoaminooxidasa tipo B (iMAO-B)}

La selegilina y rasagilina inhiben de manera irreversible la MAO-B, mientras que la safinamida es un inhibidor selectivo y reversible de la MAO-B, ambos mecanismos evitan la degradación de la dopamina. Estos medicamentos han sido usados en el tratamiento sintomático inicial de la EP, pero la mejoría en los síntomas solo es leve por lo que termina siendo necesario 
asociarlos a otros medicamentos ${ }^{77}$. Los iMAO-B generan pocos efectos adversos, pueden mejorar la calidad de vida, retrasan la necesidad de levodopa y, en el caso de la rasagilina, podrían estar asociados a un efecto neuroprotector ${ }^{101}$. Las dosis recomendadas son: selegilina $5 \mathrm{mg} /$ día en terapia asociada a levodopa, rasagilina $1 \mathrm{mg} /$ día en monoterapia o 0,5-1 mg/día en terapia con levodopa y safinamida 50-100 mg asociada a levodopa ${ }^{77,102}$. Los efectos adversos incluyen náuseas, cefalea, mareo, hipotensión ortostática, confusión y alucinaciones $^{101}$.

\section{Amantadina}

Este medicamento con propiedades antivirales bloquea los receptorees NMDA (N-metil-D-aspartato) de glutamato, tiene acción anticolinérgica y aumenta las concentraciones de dopamina ${ }^{103}$. La amantadina logra efectos leves y transitorios en los síntomas de la EP por lo que anteriormente se usaba como monoterapia por periodos cortos, pero actualmente se suele asociar al tratamiento con levodopa cuando han aparecido fluctuaciones motoras o discinesias ${ }^{104-106}$. La dosis diaria es de $300 \mathrm{mg} /$ día dividida en tres dosis ${ }^{77}$. Los efectos adversos son cefalea, náuseas, livedo reticulares, insomnio, edema de tobillos, confusión y alucinaciones $^{77}$.

\section{Anticolinérgicos}

A este grupo pertenecen el trihexifenidilo, la benztropina y el biperideno que están indicados en los pacientes con temblor que no responde a levodopa o agonistas dopaminérgicos, y en pacientes jóvenes sin compromiso cognitivo que presentan temblor incapacitante con poca bradicinesia y rigidez ${ }^{77,107}$. Se deben empezar a dosis bajas y aumentar progresivamente para evitar los efectos adversos que son comunes en estos medicamentos (xerostomía, visión borrosa, midriasis, fotofobia, confusión y alucinaciones) principalmente en pacientes de mayor edad ${ }^{108}$. La dosis inicial de trihexifenidilo es de $1 \mathrm{mg} /$ día hasta alcanzar $6 \mathrm{mg} /$ día dividido en tres dosis; la benztropina se inicia con 0,5-1 mg/día hasta 3-6 mg/ día dividido en tres dosis; y el biperideno que es el único de estos medicamentos disponible en nuestro medio se inicia con $2 \mathrm{mg}$ dos a tres veces al día hasta $16 \mathrm{mg} /$ día $^{77}$.

\section{Inhibidores de la Catecol-O-metiltransferasa (i-COMT)}

La tolcapona y entacapona evitan el metabolismo de L-dopa a 3-O-metildopa, con lo cual aumentan la vida media plasmática de la levodopa cuando se dan simultáneamente con ésta ${ }^{109}$. Estos medicamentos se usan principalmente en el tratamiento de las fluctuaciones motoras asociadas al tratamiento con levodopa ${ }^{110}$, aunque su inicio conjunto con levodopa no retrasa la aparición o disminuye la frecuencia de discinesias $^{111}$. La dosis inicial de tolcapona es $100 \mathrm{mg}$ tres veces al día, aunque no está disponible en nuestro medio; la dosis de entacapona es de $200 \mathrm{mg}$ con cada tableta de levodopa, hasta un máximo de ocho dosis por día ${ }^{77}$. Los efectos adversos más frecuentes son las discinesias, alucinaciones, confusión, náuseas y coloración de la orina ${ }^{77}$.

\section{Tratamiento de los síntomas no motores}

Al igual que con los síntomas motores, los síntomas no motores tienen diferentes opciones de tratamiento, las cuales dependen de las comorbilidades del paciente, la gravedad de los síntomas, los medicamentos usados para tratar los síntomas motores y la aparición de complicaciones o efectos adversos derivados de éstos $^{112,113}$, Tabla 3.

\section{Tratamiento quirúrgico}

Actualmente, la estimulación cerebral profunda (ECP) es la terapia más extendida para el tratamiento quirúrgico de la EP, aunque antes de esto la talamotomía, palidotomía y subtalamotomía eran las únicas opciones que tenían los pacientes con EP avanzada, pero fueron reemplazadas por la ECP al mostrar menos complicaciones y necesidad de repetición, posibilidad de terapia bilateral y mejorías en las funciones motoras y no motoras iguales o mayores ${ }^{114,115}$. Sin embargo, las intervenciones neuroablativas podrían ser consideradas en pacientes que no responden a la ECP o han tenido complicaciones por ésta, teniendo en cuenta que son menos costosas y no necesitan el seguimiento estricto de la $\mathrm{ECP}^{116-119}$.

La ECP es una técnica de neuromodulación cerebral que se ha empleado en pacientes con EP así como para tratar la distonia, temblor esencial, depresión mayor, trastorno obsesivo compulsivo, entre otras ${ }^{120,121}$. La ECP ha demostrado su efectividad en los pacientes con EP en quienes los síntomas han progresado $\mathrm{y}$ perdido la respuesta a la terapia farmacológica, en aquellos que no toleran sus efectos adversos o cuyos síntomas como el temblor son refractarios ${ }^{122,123}$. En esta terapia de estimulación eléctrica se implanta uno o más electrodos en regiones cerebrales específicas, los cuales se conectan a un marcapaso subcutáneo implantado en el pecho desde donde se modula 
eléctricamente los patrones de despolarización, repolarización y conducción del potencial de acción de las neuronas ${ }^{124}$. Los sitios neuronales intervenidos con mayor frecuencia son el NST, GPi y núcleo ventral intermedio del tálamo ${ }^{125}$, con lo cual se mejora la iniciación del movimiento, capacidades verbales, distonia, marcha, estabilidad postural, deglución, temblor, tiempo libre sin discinesias, entre otros, principalmente con la estimulación bilateral ${ }^{126-132}$. A pesar de que se ha descrito cambios comportamentales o cognitivos atribuibles a la $\mathrm{ECP}^{133-136}$, los efectos adversos como la rigidez postoperatoria, convulsiones parciales, eventos cerebrovasculares, trombosis venosa profunda, migración del dispositivo o lesiones causadas por las partes del dispositivo se presentan con poca frecuencia ${ }^{137-142}$. Entre los criterios para seleccionar a los pacientes que se podrían beneficiar de la ECP se encuentra EP de tipo idiopático, ausencia de alteraciones cognitivas o psiquiátricas, fluctuaciones motoras y/o complicaciones intratables farmacológicamente, temblor resistente a medicamentos y buena respuesta ante la terapia dopaminérgica ${ }^{123,143,144}$.

Tabla 3. Manejo de los síntomas no motores.

\begin{tabular}{ll}
\hline Demencia & Rivastigmina, donepezil, galantamina, memantina (153-157) \\
Ansiedad & Tratar los períodos “wearing off”, benzodiazepinas, \\
Depresión & Modificar AD (117, 158) \\
Apatía & Pramipexole, ATC (159, 160) \\
& Metilfenidato, levodopa, AD (117, 161) \\
Alucinaciones y psicosis & Modificar: iMAO-B, anticolinérgicos, amantadine, AD \\
& Clozapina, quetiapina (118) \\
Hipotensión ortostática & Métodos no farmacológicos * \\
Insomnio & Midrodine, fludrocortisona (162) \\
Sialorrea & Higiene del sueño \\
Disfunción vesical & Levodopa, zolpidem, trazodona (117, 163) \\
& Anticolinérgicos (160) \\
Somnolencia diurna excesiva & Ejercicios de piso pélvico \\
TCSR & Anticolinérgicos (164) \\
Disfunción eréctil & Modafinilo \\
Dolor & Modificar o ajustar AD (117, 163) \\
& Clonazepam (117, 163) \\
& Sildenafil \\
Apomorfina (117) & Ajustar terapia, AINES, antidepresivos (117, 165) \\
&
\end{tabular}

*Medias de compresión, ingesta de agua, evitar el alcohol y fragmentar las comidas (166). AD: agonista dopaminérgico; ATC: antidepresivo tricíclico; iMAO-B: inhibidor de la monoaminooxidasa tipo B; AINES: antiinflamatorios no esteroideos; TCSR: trastorno de conducta del sueño REM.

\section{Otras terapias}

La terapia génica, terapia celular para trasplantes autólogos, trasplantes de células nigrales dopaminérgicas fetales y el uso de factores neurotrópicos son posibilidades terapéuticas que llevan ya varias décadas siendo evaluadas pero se han descrito resultados variables cuando son aplicadas en paciente con EP, razón por la cual es necesaria aún mayor experiencia clínica $^{145,146}$.

Se debe tener en cuenta también los tratamientos no farmacológicos que incluyen la terapia física, ocupacional, del habla, de la deglución, psicológica, el entrenamiento en la marcha y balance que son particularmente importantes en las fases avanzadas de la EP, razón por la cual se necesita de un equipo interdisciplinario para lograr un abordaje integral de los pacientes con $\mathrm{EP}^{147}$.

\section{Conflicto de interés}

Ninguno por declarar.

\section{Referencias}

1. Fereshtehnejad SM, Shafieesabet M, Rahmani A, Delbari A, Lökk J. Medium-to-high prevalence of screening-detected parkinsonism in the urban area of Tehran, Iran: data from a community-based door- 
to-door study. Neuropsychiatr Dis Treat. 2015; 11: 321-332. DOI: 10.2147/NDT.S77391.

2. De Lau LM, Breteler MM. Epidemiology of Parkinson's disease. Lancet Neurol. 2006; 5(6): 525-535. DOI: 10.1016/s1474-4422(06)70471-9.

3. de Rijk Md, Tzourio C, Breteler M, Dartigues J, Amaducci L, Lopez-Pousa S, et al. Prevalence of parkinsonism and Parkinson's disease in Europe: the EUROPARKINSON Collaborative Study. European community concerted action on the epidemiology of Parkinson's disease. J Neurol Neurosurg Psychiatry. 1997; 62(1): 10-15.

4. Wirdefeldt K, Adami H-O, Cole P, Trichopoulos D, Mandel J. Epidemiology and etiology of Parkinson's disease: a review of the evidence. Eur J Epidemiol. 2011; 26(Sup 1): 1-58. doi: 10.1007/s10654-0119581-6.

5. Twelves D, Perkins KS, Counsell C. Systematic review of incidence studies of Parkinson's disease. Mov Disord. 2003; 18(1): 19-31.

6. Hirsch L, Jette N, Frolkis A, Steeves T, Pringsheim T. The incidence of Parkinson's disease: a systematic review and meta-analysis. Neuroepidemiology. 2016; 46(4): 292-300. DOI: 10.1159/000445751.

7. Barbosa MT, Caramelli P, Maia DP, Cunningham MCQ, Guerra HL, Lima-Costa MF, et al. Parkinsonism and Parkinson's disease in the elderly: A community-based survey in Brazil (the Bambuí study). Mov Disord. 2006; 21(6): 800-808. DOI: 10.1002/mds.20806.

8. Benito-Leon J, Bermejo-Pareja F, MoralesGonzalez J, Porta-Etessam J, Trincado R, Vega S, et al. Incidence of Parkinson disease and parkinsonism in three elderly populations of central Spain. Neurology. 2004; 62(5): 734-741.

9. Van Den Eeden SK, Tanner CM, Bernstein AL, Fross RD, Leimpeter A, Bloch DA, et al. Incidence of Parkinson's disease: variation by age, gender, and race/ethnicity. Am J Epidemiol. 2003; 157(11): 1015-1022.

10. Pradilla A, Vesga A, Boris E, León-Sarmiento FE, Roselli DA, Bautista LE, et al. Estudio neuroepidemiológico nacional (EPINEURO) colombiano. 2003; 14(1): 104-111

11. Wooten G, Currie L, Bovbjerg V, Lee J, Patrie J. Are men at greater risk for Parkinson's disease than women? J Neurol Neurosurg Psychiatry. 2004; 75(4): 637-639.

12. Das SK, Misra AK, Ray BK, Hazra A, Ghosal MK, Chaudhuri A, et al. Epidemiology of Parkinson disease in the city of Kolkata, India: A communitybased study. Neurology. 2010; 75(15): 1362-1369. DOI: 10.1212/WNL.0b013e3181f735a 7.
13. Duarte J, García Olmos LM, Mendoza A, Clavería LE. The natural history of Parkinson's disease in the province of Segovia: mortality in a longitudinal study (20-year follow-up). Acta Neurol Scand. 2013; 127(5): 295-300. DOI: 10.1111/ane.12003.

14. Forsaa E, Larsen J, Wentzel-Larsen T, Alves G. What predicts mortality in Parkinson disease? A prospective population-based long-term study. Neurology. 2010; 75(14): 1270-1276. doi: 10.1212/ WNL.0b013e3181f61311.

15. Fernandes GC, Socal MP, Schuh AFS, Rieder CRM. Clinical and Epidemiological Factors Associated with Mortality in Parkinson's Disease in a Brazilian Cohort. Parkinson's Disease. 2015; 1(1): 1-6. DOI: 10.1155/2015/959304.

16. Pennington S, Snell K, Lee M, Walker R. The cause of death in idiopathic Parkinson's disease. Parkinsonis Relat Disord. 2010; 16(7): 434-437. DOI: 10.1016/j.parkreldis.2010.04.010.

17. Priyadarshi A, Khuder SA, Schaub EA, Priyadarshi SS. Environmental risk factors and Parkinson's disease: a metaanalysis. Environ Res. 2001; 86(2): 122-127. DOI: 10.1006/enrs.2001.4264

18. Ishihara LS, Cheesbrough A, Brayne C, Schrag A. Estimated life expectancy of Parkinson's patients compared with the UK population. J Neurol Neurosurg Psychiatry. 2007; 78(12): 1304-1309.

19. Yoritaka A, Fukae J, Hatano T, Oda E, Hattori N. The Direct Cost of Parkinson Disease at Juntendo Medical University Hospital, Japan. Intern Med. 2016; 55(2): 113-119. DOI: 10.2169/ internalmedicine.55.4484.

20. Findley LJ. The economic impact of Parkinson's disease. Parkinsonism Relat disord. 2007; 13 (Sup 1): 8-12.

21. Rodríguez-Violante M, Camacho-Ordoñez A, Cervantes-Arriaga A, González-Latapí P, VelázquezOsuna S. Factores asociados a la calidad de vida de sujetos con enfermedad de Parkinson y a la carga en el cuidador. Neurologia. 2015; 30(5): 257-263. DOI: https://doi.org/10.1016/j.nrl.2014.01.008.

22. Leiknes I, Tysnes OB, Aarsland D, Larsen J. Caregiver distress associated with neuropsychiatric problems in patients with early Parkinson's disease: the Norwegian ParkWest study. Acta Neurol Scand. 2010;122(6): 418-424. DOI: 10.1111/j.16000404.2010.01332.x.

23. Forsaa EB, Larsen JP, Wentzel-Larsen T, Herlofson K, Alves G. Predictors and course of health-related quality of life in Parkinson's disease. Mov Disord. 2008; 23(10): 1420-1427. DOI: 10.1002/mds.22121.

24. Rodríguez-Violante M, Cervantes-Arriaga A, Berlanga-Flores C, Ruiz-Chow A. Depresión como 
determinante de la calidad de vida de pacientes mexicanos con enfermedad de Parkinson. Arch Neurocien Mex. 2011; 16 (Sup 1): 120-22.

25. Gómez-Esteban JC, Tijero B, Somme J, Ciordia R, Berganzo K, Rouco I, et al. Impact of psychiatric symptoms and sleep disorders on the quality of life of patients with Parkinson's disease. J Neurol. 2011; 258(3): 494-499. doi: 10.1007/s00415-010-5786-y.

26. Hanna KK, Cronin-Golomb A. Impact of anxiety on quality of life in Parkinson's disease. Parkinsons Dis. 2012; 1(1): 1-8. DOI: 10.1155/2012/640707.

27. Fumadó JC. Enfermedad de Parkinson y enfermedad de Alzheimer: factores de riesgo ambientales. Neurología. 2014; 29(9): 541-549. DOI: https://doi. org/10.1016/j.nrl.2012.04.001.

28. Valdés EG, Andel R, Sieurin J, Feldman AL, Edwards JD, Langström N, et al. Occupational complexity and risk of Parkinson's disease. PloS one. 2014; 9(9): e106676. DOI: 10.1371/journal. pone. 0106676 .

29. Dick FD, De Palma G, Ahmadi A, Scott N, Prescott G, Bennett J, et al. Environmental risk factors for Parkinson's disease and parkinsonism: the Geoparkinson study. Occup Environ Med. 2007; 64(10): 666-672.

30. McCormack AL, Thiruchelvam M, ManningBog AB, Thiffault C, Langston JW, CorySlechta DA, et al. Environmental risk factors and Parkinson's disease: selective degeneration of nigral dopaminergic neurons caused by the herbicide paraquat. Neurobiol Dis. 2002; 10(2): 119-127.

31. Noyce AJ, Bestwick JP, Silveira-Moriyama L, Hawkes CH, Giovannoni G, Lees AJ, et al. Metaanalysis of early nonmotor features and risk factors for Parkinson disease. Ann Neurol. 2012; 72(6): 893-901. DOI: 10.1002/ana.23687.

32. Lill CM. Genetics of Parkinson's disease. Molecul Cellular Probes. 2016; 30(6): 386-396. DOI: https:// doi.org/10.1016/j.mcp.2016.11.001.

33. Satake W, Nakabayashi Y, Mizuta I, Hirota Y, Ito C, Kubo M, et al. Genome-wide association study identifies common variants at four loci as genetic risk factors for Parkinson's disease. Nat Genet. 2009; 41(12): 1303-1307. DOI: 10.1038/ng.485.

34. Alves G, Müller B, Herlofson K, HogenEsch I, Telstad W, Aarsland D, et al. Incidence of Parkinson's disease in Norway: the Norwegian ParkWest study. J Neurol Neurosurg Psychiatry. 2009; 80(8): 851-857.

35. Michel PP, Hirsch EC, Hunot S. Understanding Dopaminergic Cell Death Pathways in Parkinson Disease. Neuron. 2016; 90(4): 675-691. DOI: 10.1016/j.neuron.2016.03.038.
36. Abou-Sleiman PM, Muqit MM, Wood NW. Expanding insights of mitochondrial dysfunction in Parkinson's disease. Nat Rev Neurosci. 2006; 7(3): 207-19. DOI: $10.1038 / \mathrm{nrn} 1868$.

37. Schapira AHV, Cooper JM, Dexter D, Clark JB, Jenner P, Marsden CD. Mitochondrial Complex I Deficiency in Parkinson's Disease. J Neurochem. 1990; 54(3): 823-827.

38. Langston JW, Ballard P, Tetrud JW, Irwin I. Chronic Parkinsonism in humans due to a product of meperidine-analog synthesis. Science. 1983; 219(4587): 979-980.

39. Langston JW, Forno LS, Rebert CS, Irwin I. Selective nigral toxicity after systemic administration of 1-methyl-4-phenyl-1,2,5,6-tetrahydropyrine (MPTP) in the squirrel monkey. Brain Res. 1984; 292(2): 390-394.

40. Palacino JJ, Sagi D, Goldberg MS, Krauss S, Motz C, Wacker M, et al. Mitochondrial Dysfunction and Oxidative Damage in parkin-deficient Mice. J Biol Chemistry. 2004; 279(18): 18614-18622. DOI: 10.1074/jbc.M401135200.

41. Gautier CA, Kitada T, Shen J. Loss of PINK1 causes mitochondrial functional defects and increased sensitivity to oxidative stress. Proc Nati Acad Sci USA. 2008; 105(32): 11364-11369. DOI: 10.1073/ pnas.0802076105.

42. Deas E, Wood NW, Plun-Favreau H. Mitophagy and Parkinson's disease: The PINK1-parkin link. Biochim Biophys Acta. 2011; 1813(4): 623-633. DOI: $10.1016 /$ j.bbamcr.2010.08.007.

43. Burré J, Sharma M, Tsetsenis T, Buchman V, Etherton MR, Südhof TC. Alpha-Synuclein Promotes SNARE-complex assembly in vivo and in vitro. Science. 2010; 329(5999): 1663-1667. DOI: 10.1126/science. 1195227 .

44. Polymeropoulos MH, Lavedan C, Leroy E, Ide SE, Dehejia A, Dutra A, et al. Mutation in the $\alpha$-Synuclein Gene Identified in Families with Parkinson's Disease. Science. 1997; 276(5321): 2045-2047.

45. Spillantini MG, Schmidt ML, Lee VMY, Trojanowski JQ, Jakes R, Goedert M. $\alpha$-Synuclein in Lewy bodies. Nature. 1997; 388(6645): 839-840.

46. Lashuel HA, Overk CR, Oueslati A, Masliah E. The many faces of $\alpha$-synuclein: from structure and toxicity to therapeutic target. Nat Rev Neurosci. 2013; 14(1): 38-48.

47. Luk KC, Kehm V, Carroll J, Zhang B, O’Brien P, Trojanowski JQ, et al. Pathological $\alpha$-synuclein transmission initiates Parkinsonlike neurodegeneration in nontransgenic mice. Science. 2012; 338(6109): 949-953. DOI: 10.1126/ 
science. 1227157.

48. Xilouri M, Brekk OR, Stefanis L. Autophagy and Alpha-Synuclein: Relevance to Parkinson's Disease and Related Synucleopathies. Mov Disord. 2016; 31(2): 178-192. DOI: 10.1002/mds.26477.

49. Chu Y, Dodiya H, Aebischer P, Olanow CW, Kordower JH. Alterations in lysosomal and proteasomal markers in Parkinson's disease: Relationship to alpha-synuclein inclusions. Neurobiol Dis. 2009; 35(3): 385-398. doi: 10.1016/j. nbd.2009.05.023.

50. Winslow AR, Chen C-W, Corrochano S, AcevedoArozena A, Gordon DE, Peden AA, et al. $\alpha$-Synuclein impairs macroautophagy: implications for Parkinson's disease. J Cell Biol. 2010; 190(6): 1023-1037.

51. Cuervo AM, Stefanis L, Fredenburg R, Lansbury PT, Sulzer D. Impaired Degradation of Mutant $\alpha$-Synuclein by Chaperone-Mediated Autophagy. Science. 2004; 305(5688): 1292-1295.

52. Sánchez JC, López-Zapata DF, RomeroLeguizamón CR. Mecanismos de transporte de calcio en neuroprotección y neurotoxicidad. Rev Neurol. 2010; 51(10): 624-632.

53. Damier P, Hirsch EC, Agid Y, Graybiel AM. The substantia nigra of the human brain. II Patterns of loss of dopamine-containing neurons in Parkinson's disease. Brain. 1999; 122(8): 1437-1448.

54. Kish SJ, Shannak K, Hornykiewicz O. Uneven pattern of dopamine loss in the striatum of patients with idiopathic Parkinson's disease. N Engl J Med. 1988; 318(14): 876-880. DOI: 10.1056/ NEJM198804073181402.

55. DeLong MR, Wichmann T. Circuits and circuit disorders of the basal ganglia. Arch Neurol. 2007; 64(1): 20-24. DOI: 10.1001/archneur.64.1.20.

56. Calabresi P, Picconi B, Tozzi A, Ghiglieri V, Di Filippo M. Direct and indirect pathways of basal ganglia: a critical reappraisal. Nat Neurosci. 2014; 17(8): 1022-1030.

57. Gerfen CR, Surmeier DJ. Modulation of striatal projection systems by dopamine. Annual review of neuroscience. 2011; 34: 441-66.

58. Gelb DJ, Oliver E, Gilman S. Diagnostic criteria for Parkinson disease. Arc Neurol. 1999; 56(1): 33-39.

59. Hughes AJ, Daniel SE, Kilford L, Lees AJ. Accuracy of clinical diagnosis of idiopathic Parkinson's disease: a clinico-pathological study of 100 cases. J Neurol Neurosurg Psychiatry. 1992; 55(3): 181-184.

60. Rodriguez-Oroz MC, Jahanshahi M, Krack P, Litvan I, Macias R, Bezard E, et al. Initial clinical manifestations of Parkinson's disease: features and pathophysiological mechanisms. Lancet Neurol.
2009; 8(12): 1128-1139. DOI: 10.1016/S14744422(09)70293-5.

61. Berardelli A, Rothwell JC, Thompson PD, Hallett M. Pathophysiology of bradykinesia in Parkinson's disease. Brain. 2001; 124(11): 2131-2146. DOI: https://doi.org/10.1093/brain/124.11.2131.

62. Jankovic J. Parkinson's disease: clinical features and diagnosis. J Neurol Neurosurg Psychiatry. 2008; 79(4): 368-376. DOI: 10.1136/jnnp.2007.131045.

63. Massano J, Bhatia KP. Clinical approach to Parkinson's disease: features, diagnosis, and principles of management. Cold Spring Harb Perspect Med. 2012; 2(6): a008870. DOI: 10.1101/ cshperspect.a008870.

64. Sveinbjornsdottir S. The clinical symptoms of Parkinson's disease. J Neurochem. 2016; 139 (Sup. 1): 318-324. DOI: 10.1111/jnc. 13691.

65. Belvisi D, Conte A, Bologna M, Bloise MC, Suppa A, Formica A, et al. Re-emergent tremor in Parkinson's disease. Parkinsonism Relat Disord. 2017; 36(1): 41-46. DOI: 10.1016/j.parkreldis.2016.12.012.

66. Broussolle E, Krack P, Thobois S, Xie-Brustolin J, Pollak P, Goetz CG. Contribution of Jules Froment to the study of parkinsonian rigidity. Mov Disorders. 2007; 22(7): 909-914. DOI: 10.1002/mds.21484.

67. Williams D, Watt H, Lees A. Predictors of falls and fractures in bradykinetic rigid syndromes: a retrospective study. J Neurol Neurosurg Psychiatry. 2006; 77(4): 468-473. DOI: 10.1136/ jnnp.2005.074070.

68. Braak H, Del Tredici K, Rüb U, De Vos RA, Steur ENJ, Braak E. Staging of brain pathology related to sporadic Parkinson's disease. Neurobiology of aging. 2003; 24(2): 197-211.

69. Braak H, Rüb U, Steur EJ, Del Tredici K, De Vos R. Cognitive status correlates with neuropathologic stage in Parkinson disease. Neurology. 2005; 64(8): 1404-1410. DOI: 10.1212/01. WNL.0000158422.41380.82.

70. Chaudhuri KR, Healy DG, Schapira AH. Nonmotor symptoms of Parkinson's disease: diagnosis and management. Lancet Neurol. 2006; 5(3): 235 245. DOI: $10.1016 / \mathrm{S} 1474-4422(06) 70373-8$.

71. Garcia-Borreguero D, Larrosa O, Bravo M. Parkinson's disease and sleep. Sleep Med Rev. 2003; 7(2): 115-129. DOI: https://doi. org/10.1053/smrv.2002.0229.

72. Magerkurth C, Schnitzer R, Braune S. Symptoms of autonomic failure in Parkinson's disease: prevalence and impact on daily life. Clin Auton Res. 2005; 15(2): 76-82. DOI: 10.1007/s10286-005-0253-z.

73. Pfeiffer RF. Gastrointestinal dysfunction in Parkinson's disease. Parkinsonism Relate 
Disord. 2011; 17(1): 10-15. DOI: $10.1016 / \mathrm{j}$. parkreldis.2010.08.003.

74. Thanvi B, Munshi S, Vijaykumar N, Lo T. Neuropsychiatric non-motor aspects of Parkinson's disease. Postgrad Med J. 2003; 79(936): 561-565. DOI: $10.1136 / \mathrm{pmj} .79 .936 .561$.

75. Giuffrida R, Vingerhoets F, Bogousslavsky J, Ghika J. Pain in Parkinson's disease. Rev Neurologique. 2005; 161(4): 407-418. DOI: 10.1016/S00353787(05)85070-2.

76. Hoehn MM, Yahr MD. Parkinsonism onset, progression, and mortality. Neurology. 1967; 17(5): 427-442.

77. Connolly BS, Lang AE. Pharmacological treatment of Parkinson disease: a review. JAMA. 2014; 311(16): 1670-1683. DOI: 10.1001/jama.2014.3654.

78. Goetz CG, Pal G. Initial management of Parkinson's disease. women. 2014; 9(1): 1-11. DOI: https://doi. org/10.1136/bmj.g6258

79. Ahlskog JE. Seniors with Parkinson's Disease: Initial Medical Treatment. J Clinic Neurol. 2010; 6(4): 159-166. DOI: $10.3988 /$ jcn.2010.6.4.159.

80. Chondrogiorgi M, Tatsioni A, Reichmann H, Konitsiotis S. Dopamine agonist monotherapy in Parkinson's disease and potential risk factors for dyskinesia: a meta-analysis of levodopa-controlled trials. Eur J Neurol. 2014; 21(3): 433-440. doi: 10.1111/ene. 12318 .

81. Adler $\mathrm{CH}$, Sethi KD, Hauser R, Davis T, Hammerstad J, Bertoni J, et al. Ropinirole for the treatment of early Parkinson's disease. Neurology. 1997; 49(2): 393-399.

82. Shannon K, Bennett J, Friedman J, Group PS. Efficacy of pramipexole, a novel dopamine agonist, as monotherapy in mild to moderate Parkinson's disease. Neurology. 1997; 49(3): 724-728.

83. Talati R, Baker W, Patel A, Reinhart K, Coleman C. Adding a dopamine agonist to preexisting levodopa therapy vs. levodopa therapy alone in advanced Parkinson's disease: a meta-analysis. Int J Clin Practice. 2009; 63(4): 613-623. DOI: 10.1111/j.1742-1241.2009.02027.x.

84. Giugni JC, Okun MS. Treatment of advanced Parkinson's disease. Current Opinion Neurol. 2014; 27(4): 450-460. DOI: $10.1097 /$ WCO.0000000000000118.

85. LeWitt PA, Fahn S. Levodopa therapy for Parkinson disease A look backward and forward. Neurology. 2016; 86(14 Sup. 1): 3-12.

86. Group PS. Levodopa and the progression of Parkinson's disease. N Engl J Med. 2004; 351(24): 2498-2508. DOI: 10.1056/NEJMoa033447.

87. Manson A, Stirpe P, Schrag A. Levodopa-induced- dyskinesias clinical features, incidence, risk factors, management and impact on quality of life. J Parkinsons Dis. 2012; 2(3): 189-198. doi: 10.3233/ JPD-2012-120103.

88. Schrag A, Quinn N. Dyskinesias and motor fluctuations in Parkinson's disease: A communitybased study. Brain. 2000; 123(11): 2297-2305.

89. Chase TN, Mouradian MM, Engber TM. Motor response complications and the function of striatal efferent systems. Neurology. 1993; 43(12 Sup. 6): 23-27.

90. Aquino CC, Fox SH. Clinical spectrum of levodopainduced complications. Mov Disord. 2015; 30(1): 80-89. DOI: $10.1002 / \mathrm{mds} .26125$.

91. Calabresi P, Di Filippo M, Ghiglieri V, Tambasco N, Picconi B. Levodopa-induced dyskinesias in patients with Parkinson's disease: filling the benchto-bedside gap. Lancet Neurol. 2010; 9(11): 11061117. doi: 10.1016/S1474-4422(10)70218-0.

92. Fahn S, Bressman SB. Should levodopa therapy for parkinsonism be started early or late? Evidence against early treatment. Can J Neurol Sci. 1984; 11(Sup. 1): 200-205.

93. Müller T. Detoxification and antioxidative therapy for levodopa-induced neurodegeneration in Parkinson's disease. Expert Rev Neurother. 2013; 13(6): 707-718. DOI: 10.1586/ern.13.50.

94. Schapira AH. The clinical relevance of levodopa toxicity in the treatment of Parkinson's disease. Mov Disord. 2008; 23(Sup. 3). 515-20. DOI: 10.1002/ mds.22146.

95. Hisahara S, Shimohama S. Dopamine receptors and Parkinson's disease. International J Med Chem. 2011; 1(1): 1-16. DOI:10.1155/2011/403039.

96. Rascol O, Brooks DJ, Korczyn AD, De Deyn PP, Clarke CE, Lang AE. A five-year study of the incidence of dyskinesia in patients with early Parkinson's disease who were treated with ropinirole or levodopa. N Engl J Med. 2000; 342(20): 14841491. DOI: 10.1056/NEJM200005183422004.

97. Constantinescu R, Romer M, McDermott MP, Kamp C, Kieburtz K. Impact of pramipexole on the onset of levodopa-related dyskinesias. Mov Disord. 2007; 22(9): 1317-1319. DOI: 10.1002/mds.21292.

98. Stowe R, Ives N, Clarke C, Van Hilten J, Ferreira J, Hawker R, et al. Dopamine agonist therapy in early Parkinson's disease. Cochrane Database Syst Rev. 2008; 2(CD006564): 1-92. DOI: 10.1002/14651858. CD006564.pub2.

99. Wood LD. Clinical review and treatment of select adverse effects of dopamine receptor agonists in Parkinson's disease. Drugs Aging. 2010; 27(4): 295310. DOI: 10.2165/11318330-000000000-00000. 
100. Weintraub D, Koester J, Potenza MN, et al. Impulse control disorders in Parkinson disease: a crosssectional study of 3090 patients. Arch Neurol. 2010; 67(5): 589-595. DOI: 10.1001/archneurol.2010.65.

101.Riederer P, Laux G. MAO-inhibitors in Parkinson's Disease. Exp Neurobiol. 2011; 20(1): 1-17. DOI: 10.5607/en.2011.20.1.1.

102. Schapira A, Fox S, Hauser R, Jankovic J, Jost W, Kulisevsky J, et al. Safinamide Add on to L-Dopa: a randomized, placebo-controlled, 24-week global trial in patients with Parkinson's Disease (PD) and motor fluctuations (SETTLE)(P01. 062). Neurology. 2013; 80(Sup. 7): 1-62.

103.Mizoguchi K, Yokoo H, Yoshida M, Tanaka T, Tanaka M. Amantadine increases the extracellular dopamine levels in the striatum by re-uptake inhibition and by N-methyl--aspartate antagonism. Brain Res. 1994; 662(1-2): 255-258.

104.Wolf E, Seppi K, Katzenschlager R, Hochschorner G, Ransmayr G, Schwingenschuh P, et al. Long-term antidyskinetic efficacy of amantadine in Parkinson's disease. Mov Disord. 2010; 25(10): 1357-1363. DOI: $10.1002 / \mathrm{mds} .23034$.

105. Ory-Magne F, Corvol J-C, Azulay J-P, Bonnet A-M, Brefel-Courbon C, Damier P, et al. Withdrawing amantadine in dyskinetic patients with Parkinson disease The AMANDYSK trial. Neurology. 2014; 82(4): 300-307.

106.Pappa S, Tsouli S, Apostolou G, Mavreas V, Konitsiotis S. Effects of Amantadine on Tardive Dyskinesia: A Randomized, Double-Blind, PlaceboControlled Study. Clin Neuropharmacol. 2010; 33(6): 271-275.

107.Friedman J, Koller W, Lannon M, Busenbark K, Swanson-Hyland E, Smith D. Benztropine versus clozapine for the treatment of tremor in Parkinson's disease. Neurology. 1997; 48(4): 1077-1080.

108.Katzenschlager R, Sampaio C, Costa J, Lees A. Anticholinergics for symptomatic management of Parkinson s disease. Cochrane Database Syst Rev. 2002; 3(CD003735): 1-22. DOI: 10.1002/14651858. CD003735.

109.Keränen T, Gordin A, Harjola V, Karlsson M, Korpela K, Pentikäinen $\mathrm{P}$, et al. The effect of catechol-O-methyl transferase inhibition by entacapone on the pharmacokinetics and metabolism of levodopa in healthy volunteers. Clin Neuropharmacol. 1993; 16(2): 145-156.

110.Rinne U, Larsen J, Siden Å, Worm-Petersen J. Entacapone enhances the response to levodopa in parkinsonian patients with motor fluctuations. Neurology. 1998; 51(5): 1309-1314.

111.Stocchi F, Rascol O, Kieburtz K, Poewe W,
Jankovic J, Tolosa E, et al. Initiating levodopa/ carbidopa therapy with and without entacapone in early Parkinson disease: the STRIDE-PD study. Ann Neurol. 2010; 68(1): 18-27. DOI: 10.1002/ ana. 22060 .

112.Chaudhuri KR, Schapira AH. Non-motor symptoms of Parkinson's disease: dopaminergic pathophysiology and treatment. Lancet Neurol. 2009; 8(5): 464-474. DOI: 10.1016/S14744422(09)70068-7.

113.Seppi K, Weintraub D, Coelho M, Perez-Lloret S, Fox SH, Katzenschlager R, et al. The Movement Disorder Society Evidence-Based Medicine Review Update: Treatments for the non-motor symptoms of Parkinson's disease. Mov Disord. 2011; 26(Sup. 3): 42-80. DOI: 10.1002/mds.23884.

114. Tasker RR. Deep brain stimulation is preferable to thalamotomy for tremor suppression. Surg Neurol. 1998; 49(2): 145-153.

115.Esselink R, De Bie R, De Haan R, Lenders M, Nijssen P, Staal M, et al. Unilateral pallidotomy versus bilateral subthalamic nucleus stimulation in PD A randomized trial. Neurology. 2004; 62(2): 201-207.

116.Spindola B, Leite MA, Orsini M, Fonoff E, Landeiro JA, Pessoa BL. Ablative surgery for Parkinson's disease: Is there still a role for pallidotomy in the deep brain stimulation era? Clin Neurol Neurosurg. 2017; 158: 33-39. doi: 10.1016/j. clineuro.2017.04.018.

117.Hariz MI, Hariz G-M. Therapeutic stimulation versus ablation. In: Lozano AM, Hallett M, eds. Handbook of Clinical Neurology: Elsevier, 2013. p. 63-71.

118.Bulluss KJ, Pereira EA, Joint C, Aziz TZ. Pallidotomy after chronic deep brain stimulation. Neurosurg Focus. 2013; 35(5): 1-4. DOI: 10.3171/2013.8.FOCUS13293.

119.Bahgat D, Magill ST, Berk C, McCartney S, Burchiel KJ. Thalamotomy as a treatment option for tremor after ineffective deep brain stimulation. Stereotact Funct Neurosurg. 2013; 91(1): 18-23. doi: 10.1159/000342491.

120.Miocinovic S, Somayajula S, Chitnis S, Vitek JL. History, applications, and mechanisms of deep brain stimulation. JAMA Neurol. 2013; 70(2): 163-171. doi: 10.1001/2013.jamaneurol.45.

121.Goodman WK, Alterman RL. Deep Brain Stimulation for Intractable Psychiatric Disorders. Ann Rev Med. 2012; 63(1): 511-524. doi: 10.1146/ annurev-med-052209-100401

122.Fox SH, Katzenschlager R, Lim SY, Ravina B, Seppi K, Coelho M, et al. The Movement Disorder 
Society Evidence-Based Medicine Review Update: Treatments for the motor symptoms of Parkinson's disease. Mov Disord. 2011; 26(Sup. 3): S2-41. DOI: $10.1002 / \mathrm{mds} .23829$.

123.Okun MS. Deep-Brain Stimulation for Parkinson's Disease. N Engl J Med. 2012; 367(16): 1529-1538.

124.Hickey P, Stacy M. Deep Brain Stimulation: A Paradigm Shifting Approach to Treat Parkinson's Disease. Front Neurosci. 2016; 10 (173): 1-11. DOI: doi.org/10.3389/fnins.2016.00173.

125.DeLong MR, Wichmann T. Basal Ganglia Circuits as Targets for Neuromodulation in Parkinson Disease. JAMA Neurol. 2015; 72(11): 1354-1360. DOI: 10.1001/jamaneurol.2015.2397.

126.Kahan J, Urner M, Moran R, Flandin G, Marreiros A, Mancini L, et al. Resting state functional MRI in Parkinson's disease: the impact of deep brain stimulation on 'effective' connectivity. Brain. 2014; 137(4): 1130-1144.

127.Kalbe E, Voges J, Weber T, Haarer M, Baudrexel S, Klein JC, et al. Frontal FDG-PET activity correlates with cognitive outcome after STN-DBS in Parkinson disease. Neurology. 2009; 72(1): 42-49.

128.Follett KA, Weaver FM, Stern M, Hur K, Harris CL, Luo P, et al. Pallidal versus subthalamic deepbrain stimulation for Parkinson's disease. N Engl J Med. 2010; 362(22): 2077-2091.

129.Mure H, Hirano S, Tang CC, Isaias IU, Antonini A, Ma Y, et al. Parkinson's disease tremorrelated metabolic network: characterization, progression, and treatment effects. Neuroimage. 2011; 54(2): 1244-1253. DOI: $10.1016 / \mathrm{j}$. neuroimage.2010.09.028.

130.Pandey S, Sarma N. Deep brain stimulation: current status. Neurology India. 2015; 63(1): 9-18.

131.Deuschl G, Schade-Brittinger C, Krack P, Volkmann J, Schafer H, Botzel K, et al. A randomized trial of deep-brain stimulation for Parkinson's disease. N Engl J Med. 2006; 355(9): 896-908. DOI: 10.1056/ NEJMoa060281.

132. Weaver FM, Follett K, Stern M, Hur K, Harris C, Marks WJ, et al. Bilateral deep brain stimulation vs best medical therapy for patients with advanced Parkinson disease: a randomized controlled trial. JAMA. 2009; 301(1): 63-73. DOI: 10.1001/ jama.2008.929.

133.Kim H-J, Jeon BS, Yun JY, Kim YE, Yang H-J, Paek SH. Initial cognitive dip after subthalamic deep brain stimulation in Parkinson disease. J Neurol. 2013; 260(8): 2130-2133. DOI: 10.1007/ s00415-013-6959-2.

134.Rothlind JC, York MK, Carlson K, Luo P, Marks WJ, Jr., Weaver FM, et al. Neuropsychological changes following deep brain stimulation surgery for Parkinson's disease: comparisons of treatment at pallidal and subthalamic targets versus best medical therapy. J Neurol Neurosurg Psychiatry. 2015; 86(6): 622-629. DOI: 10.1136/jnnp-2014-308119.

135.Odekerken VJ, Boel JA, Geurtsen GJ, Schmand BA, Dekker IP, de Haan RJ, et al. Neuropsychological outcome after deep brain stimulation for Parkinson disease. Neurology. 2015; 84(13): 1355-1361.

136.Ashkan K, Rogers P, Bergman H, Ughratdar I. Insights into the mechanisms of deep brain stimulation. Nat Rev Neurol. 2017; 13(9): 548-54. DOI: 10.1038/nrneurol.2017.105.

137.Bannier S, Montaurier C, Derost PP, Ulla M, Lemaire J-J, Boirie Y, et al. Overweight after deep brain stimulation of the subthalamic nucleus in Parkinson disease: long term follow-up. J Neurol Neurosurg Psychiatry. 2009; 80(5): 484-488. DOI: 10.1136/jnnp.2008.158576.

138.Mandat T, Tykocki T, Koziara H, Koziorowski D, Brodacki B, Rola R, et al. Subthalamic deep brain stimulation for the treatment of Parkinson disease. Neurol Neurochir Pol. 2011; 45(1): 32-36.

139.Sharma A, Szeto K, Desilets AR. Efficacy and safety of deep brain stimulation as an adjunct to pharmacotherapy for the treatment of Parkinson disease. Ann Pharmacother. 2012; 46(2): 248-254. DOI: 10.1345/aph.1Q508.

140.Umemura A, Oka Y, Ohkita K, Yamawaki T, Yamada K. Effect of subthalamic deep brain stimulation on postural abnormality in Parkinson disease: clinical article. J Neurosurg. 2010; 112(6): 1283-1288.

141.Vergani F, Landi A, Pirillo D, Cilia R, Antonini A, Sganzerla EP. Surgical, Medical, and Hardware Adverse Events in a Series of 141 Patients Undergoing Subthalamic Deep Brain Stimulation for Parkinson Disease. World Neurosurg. 2010; 73(4): 338-344. DOI: 10.1016/j.wneu.2010.01.017.

142.Zibetti M, Rosso M, Cinquepalmi A, Lanotte M, Angrisano S, Rabbia C, et al. Asymptomatic deep venous thrombosis after deep brain stimulation for Parkinson disease. Stereotactic and functional neurosurgery. 2010; 88(2): 94-97.

143.Bronstein JM, Tagliati M,Alterman RL, LozanoAM, Volkmann J, Stefani A, et al. Deep brain stimulation for Parkinson disease: an expert consensus and review of key issues. Arch Neurol. 2011; 68(2): 165-171. DOI: 10.1001/archneurol.2010.260.

144.Moldovan A-S, Groiss SJ, Elben S, Südmeyer M, Schnitzler A, Wojtecki L. The treatment of Parkinson's disease with deep brain stimulation: 
current issues. Neural Regen Res. 2015; 10(7): 1018-1022. DOI: 10.4103/1673-5374.160094.

145.Barker RA, Drouin-Ouellet J, Parmar M. Cellbased therapies for Parkinson disease-past insights and future potential. Nat Rev Neurol. 2015; 11(9): 492-503. DOI: 10.1038/nrneurol.2015.123.

146. Yasuhara T, Kameda M, Agari T, Date I. Regenerative medicine for Parkinson's disease. Neurol Med Chir. 2015; 55(2): 113-123. DOI: 10.2176/nmc.ra.2014-0264.

147.Bloem BR, de Vries NM, Ebersbach G. Nonpharmacological treatments for patients with Parkinson's disease. Mov Disord. 2015; 30(11): 1504-1520.

148.Goetz CG, Poewe W, Rascol O, Sampaio C, Stebbins GT, Counsell C, et al. Movement Disorder Society Task Force report on the Hoehn and Yahr staging scale: status and recommendations. Mov Disord. 2004; 19(9): 1020-1028.

149.Emre M, Aarsland D, Albanese A, Byrne EJ, Deuschl G, De Deyn PP, et al. Rivastigmine for dementia associated with Parkinson's disease. N Engl J Medicine. 2004; 351(24): 2509-2518. DOI: 10.1056/NEJMoa041470.

150.Poewe W, Wolters E, Emre M, Onofrj M, Hsu C, Tekin S, et al. Long-term benefits of rivastigmine in dementia associated with Parkinson's disease: An active treatment extension study. Mov Disord. 2006; 21(4): 456-461. DOI: 10.1002/mds.20700.

151.Ravina B, Putt M, Siderowf A, Farrar JT, Gillespie M, Crawley A, et al. Donepezil for dementia in Parkinson's disease: a randomised, double blind, placebo controlled, crossover study. J Neurol Neurosurg Psychiatry. 2005; 76(7): 934-939. DOI: 10.1136/jnnp.2004.050682.

152.Litvinenko I, Odinak M, Mogil'naya V, Emelin AY. Efficacy and safety of galantamine (reminyl) for dementia in patients with Parkinson's disease (an open controlled trial). Neurosci Behav Physiol. 2008; 38(9): 937-945. DOI: 10.1007/s11055-0089077-3.

153.Emre M, Tsolaki M, Bonuccelli U, Destée A, Tolosa E, Kutzelnigg A, et al. Memantine for patients with Parkinson's disease dementia or dementia with Lewy bodies: a randomised, doubleblind, placebo-controlled trial. Lancet Neurol. 2010; 9(10): 969-977.

154.Prediger RD, Matheus FC, Schwarzbold ML, Lima MM, Vital MA. Anxiety in Parkinson's disease: a critical review of experimental and clinical studies. Neuropharmacology. 2012; 62(1): 115-124. DOI: 10.1016/j.neuropharm.2011.08.039.

155.Barone P, Poewe W, Albrecht S, Debieuvre C,
Massey D, Rascol O, et al. Pramipexole for the treatment of depressive symptoms in patients with Parkinson's disease: a randomised, double-blind, placebo-controlled trial. Lancet Neurol. 2010; 9(6): 573-580.

156.Liu J, Dong J, Wang L, Su Y, Yan P, Sun S. Comparative efficacy and acceptability of antidepressants in Parkinson's disease: a network meta-analysis. PloS one. 2013; 8(10): e76651.

157.Pagonabarraga J, Kulisevsky J, Strafella AP, Krack P. Apathy in Parkinson's disease: clinical features, neural substrates, diagnosis, and treatment. Lancet Neurol. 2015; 14(5): 518-531.

158.Mostile G, Jankovic J. Treatment of dysautonomia associated with Parkinson's disease. Parkinsonism Relat Disord. 2009; 15 (Sup. 1): 224-232. DOI: 10.1016/S1353-8020(09)70820-X.

159.Loddo G, Calandra-Buonaura G, Sambati L, Giannini G, Cecere A, Cortelli P, et al. The treatment of sleep disorders in Parkinson's disease: from research to clinical practice. Front Neurol. 2017; 8(42): 1-15. DOI: 10.3389/fneur.2017.00042.

160.Srivanitchapoom P, Pandey S, Hallett M. Drooling in Parkinson's Disease: a review. Parkinsonism Relat Disord. 2014; 20(11): 1109-1118.

161.Batla A, Tayim N, Pakzad M, Panicker JN. Treatment Options for Urogenital Dysfunction in Parkinson's Disease. Curr Treat Options Neurol. 2016; 18(45): 1-13. DOI: 10.1007/s11940-0160427-0.

162.Beiske A, Loge J, Rønningen A, Svensson E. Pain in Parkinson's disease: prevalence and characteristics. PAIN. 2009; 141(1-2): 173-7. DOI: 10.1016/j. pain.2008.12.004.

163.Sánchez-Ferro Á, Benito-León J, Gómez-Esteban JC. The management of orthostatic hypotension in Parkinson's disease. Front Neurol. 2013; 4(64): 1-11. DOI: 10.3389/fneur.2013.00064. 